Supporting Information

\title{
Flower Necklaces of Controllable Length Formed From $N$-(2-Hydroxypropyl) Methacrylamide-Based Amphiphilic Statistical Copolymers
}

Rintaro Takahashi, ${ }^{* \dagger}$ Kiyomasa Doi, ${ }^{\dagger}$ Shota Fujii, ${ }^{\dagger}$ Kazuo Sakurai ${ }^{*{ }^{*}}$

†Department of Chemistry and Biochemistry, University of Kitakyushu, 1-1, Hibikino, Wakamatsu-ku, Kitakyushu, Fukuoka, 808-0135, Japan

*(R.T.) E-Mail: r-takahashi@kitakyu-u.ac.jp, (K.S.)E-mail: sakurai@kitakyu-u.ac.jp.

\section{CONTENTS}

Preparation of precursor and P-i-Pyrj ..pp. S2

SEC-MALS measurements ..pp. S4

NMR measurements .pp. S6

Copolymer composition curve. .pp. S7

Electron density (contrast factor) pp. S8

DLS measurements ..pp. S8 
Preparation of precursor and P-i-Pyrj. The precursors were prepared by free radical polymerization of HPMA and Ma-ah-NHNH-Boc (Scheme S1) as follows: HPMA, Ma-ah-NHNH-Boc, and 2,2'-azobis(isobutyronitrile) (AIBN) (the molar ratio was 9:1:0.01) were dissolved in DMSO or methanol, in which the total solute concentration was 20 vol\%. After purging oxygen from the solution by nitrogen gas, the solution was heated to $60{ }^{\circ} \mathrm{C}$ and maintained with stirring for 20 hours under nitrogen atmosphere. The solution was poured into acetic ether (poor solvent for the polymer) to recover the polymer sample; the reprecipitation was repeated twice.

\section{Scheme S1. Chemical Reaction to Prepare Precursor}
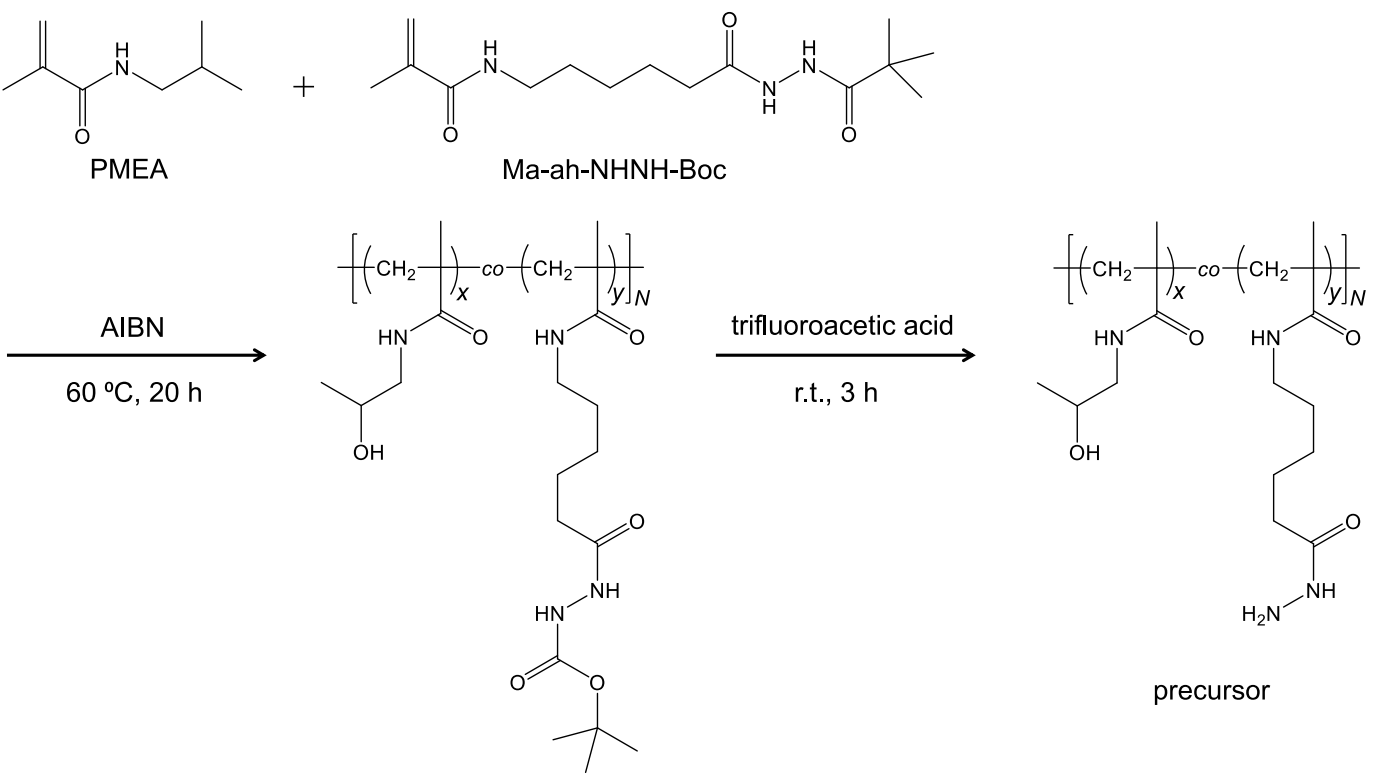

precursor

This compound and trifluoroacetic acid were mixed in 1 to 1 weight ratio in a flask, and stirred for 3 hours at room temperature. After that, the trifluoroacetic acid was removed in vacuo. Triethylamine and water were added into the flask, and then the 
solution was stirred for 1 hour at room temperature. The solution was dialyzed against water for 2 days. The obtained compound (precursor) was recovered by freeze-dry.

The precursor and 1-pyrenecarboxylic acid were dissolved in DMSO and maintained at room temperature for 6 hours with stirring. The solution was dialyzed against methanol for 3 days to remove unreacted 1-pyrenecarboxylic acid. As the ${ }^{1} \mathrm{H}$ NMR spectra for 1-pyrenecarboxylic acid and the finally-obtained compound (P-h-Pyr5 as the representative) in deuterated DMSO are shown in Fiugre S1, the signal from proton of $-\mathrm{COOH}$ at $c a .13 .5$ ppm was not observed in the ${ }^{1} \mathrm{H}$ NMR spectra of the obtained compounds. Hence, unreacted 1-pyrenecarboxylic acid could be removed (< $1 \%)$ by the dialysis.

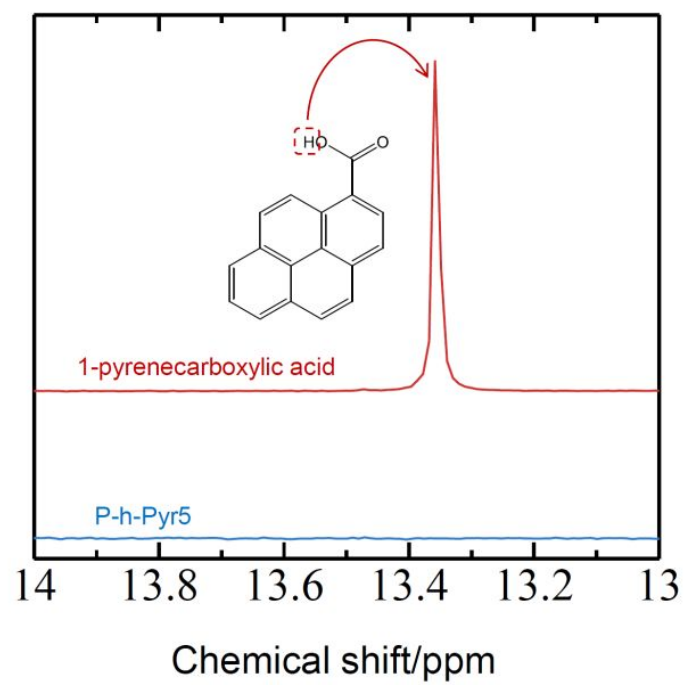

Figure S1. NMR spectra for 1-pyrenecarboxylic acid (red curve) and P-h-Pyr5 (blue curve) in deuterated DMSO. 
SEC-MALS measurements. SEC-MALS measurements were performed for the precursors using a KD-806M column (Shodex). DMAC containing $5 \mathrm{mM} \mathrm{LiBr}$ was used as eluent. The polymer concentration in the injections was $5 \mathrm{mg} / \mathrm{mL}$. Using a MALS detector (DAWN Heleous A, Wyatt Technology) with an incident light wavelength of $622.0 \mathrm{~nm}$ and a temperature maintained at $25{ }^{\circ} \mathrm{C}$, static light scattering profiles (Berry plots) were obtained to determine the molecular weight at each elusion time point (in the elution volume range where the scattering intensity is high enough). We determined the refractive index increment to be $0.0945 \mathrm{~mL} / \mathrm{g}$ by a DRM-3000 refractometer (Otsuka Electronics) at $25{ }^{\circ} \mathrm{C}$. The polymer solutions had no absorption of light at $622.0 \mathrm{~nm}$.

The fractograms for the precursors are shown in Figure S2a. The downward peaks around the elusion volume of $18 \mathrm{~mL}$ were ascribed to the solvent. We note that the monomer composition of precursor-1 (ratio of $x$ and $y$ values) were not completely equal to that of precursor-h. In addition, the polymers slightly interact with the column. Thus, the precursor-1 and precursor-h in SEC data were presumed to be not overlapped. The obtained molecular weight characteristics are listed in Table 1 in the main text.

Figure S2b shows the radius of gyration $\left(\left\langle S^{2}\right\rangle^{1 / 2}\right)$ as a function of molecular weight $(M)$ for precursor-h obtained by the SEC-MALS measurements. The solid black curve in the figure represent the fitted curves by wormlike chain model with excluded volume effect considered, given bys1

$$
\left\langle S^{2}\right\rangle^{1 / 2}=\left[1+\left(\frac{L}{3.12 l_{\mathrm{K}}}\right)^{2}+\left(\frac{L}{8.67 l_{\mathrm{K}}}\right)^{3}\right]^{\varepsilon / 3}\left\langle S^{2}\right\rangle_{0}{ }^{1 / 2}
$$




$$
\left\langle S^{2}\right\rangle_{0}=\frac{L l_{\mathrm{K}}}{6}-\frac{l_{\mathrm{K}}{ }^{2}}{4}+\frac{l_{\mathrm{K}}{ }^{3}}{4 L}-\frac{l_{\mathrm{K}}{ }^{4}}{8 L^{2}}\left[1-\exp \left(-\frac{2 L}{l_{\mathrm{K}}}\right)\right]
$$

In the fitting of the SEC-MALS data, the parameters were the molar mass per unit contour length $\left(M_{\mathrm{L}}=M / L\right), \lambda^{-1}$, and $\varepsilon$. Although $\lambda^{-1}$ could not be determined (assumed to be $1.9 \mathrm{~nm}), M_{\mathrm{L}}$ and $\varepsilon$ were almost uniquely determined to be $220 \mathrm{~nm}^{-1}$ and 0.17 , respectively. Therefore, the SEC results are reasonably described by wormlike chain model, and precursor-h is molecularly-dispersed in DMAC. Although this plot could not be made due to the low $\left\langle S^{2}\right\rangle^{1 / 2}$, we can presume that precursor-1 is also similarly dispersed in DMAC.
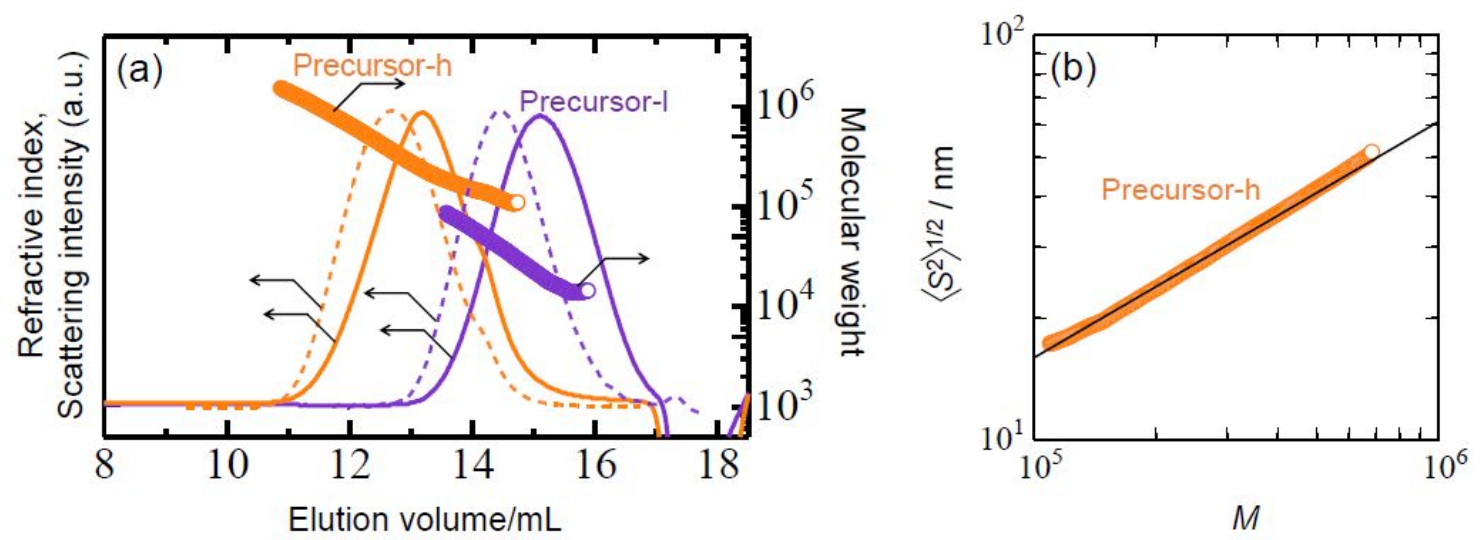

Figure S2. (a) The SEC fractograms of Precursor-1 (purple) and Precursor-h (orange).

The solid and dotted curves represent the refractive index and scattering intensity at $90^{\circ}$, respectively. (b) Radius of gyration $\left(\left\langle S^{2}\right\rangle^{1 / 2}\right)$ as a function of molecular weight $(M)$ for precursor h. The solid black curve represents the fitted curve by wormlike chain model with the excluded volume effect. 
NMR measurements. ${ }^{1} \mathrm{H}$ NMR spectroscopy was run at $25{ }^{\circ} \mathrm{C}$ using a JNM-ECP500 NMR spectrometer (JEOL) with a frequency of $500 \mathrm{MHz}$. For the solvent, deuterated dimethyl sulfoxide (containing tetramethylsilane as a standard) was used with a polymer concentration of $5 \mathrm{mg} / \mathrm{mL}$. The obtained spectra and assignment of each peak are shown in Figure S3. From the area of the peaks assigned as protons of "e", the monomer fraction ( $x$ and $y$ ) in the precursors was determined. Further, from the peaks assigned as protons of "p", "q", "r", "s", “t", and "u", the $z$ values were determined in P-i-Pyrj.
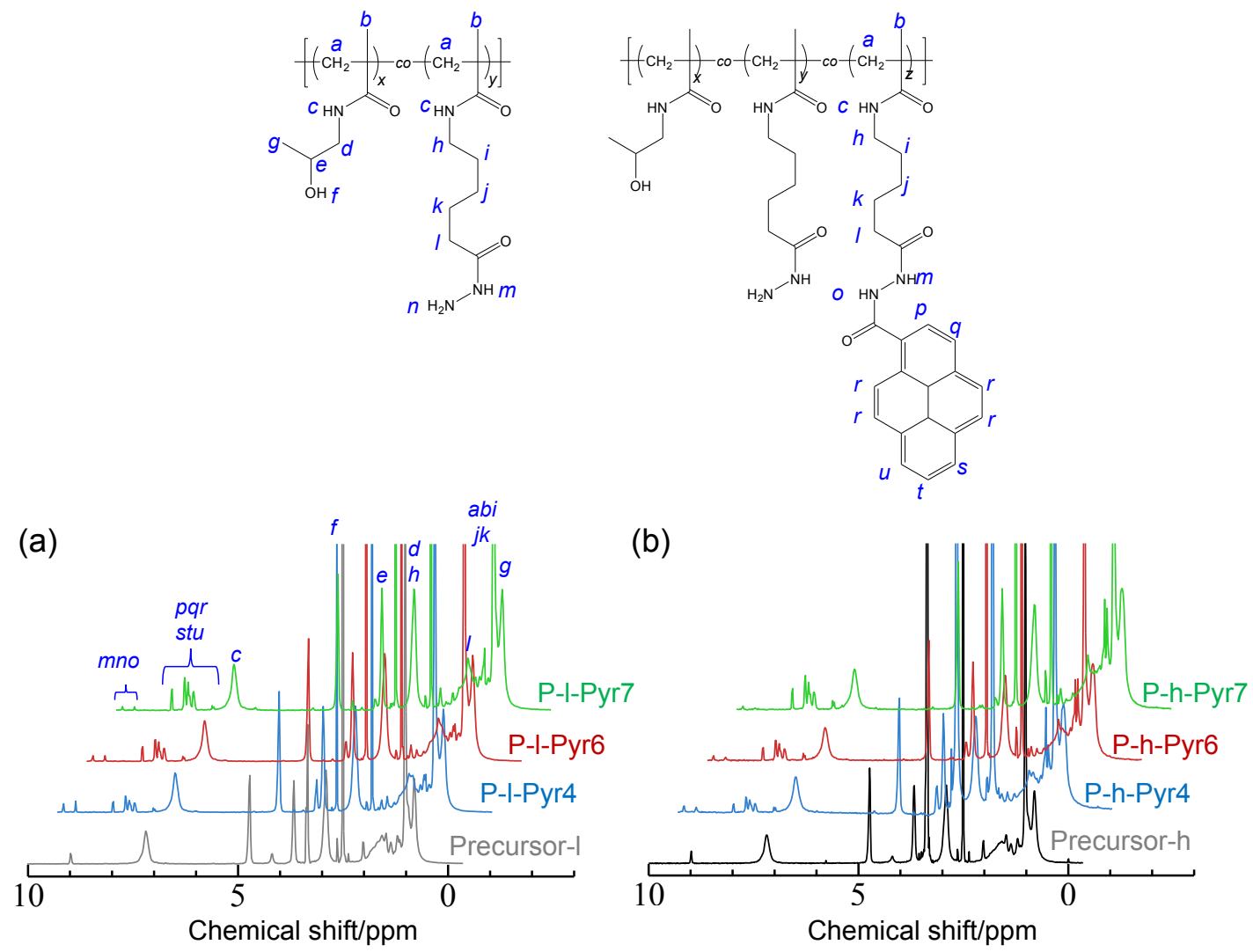

Figure S3. ${ }^{1} \mathrm{H}$ NMR spectra for Precursor- $i$ and P-i-Pyrj in deuterated DMSO at $25{ }^{\circ} \mathrm{C}$.

Panels (a) and (b) show the data for the low $N_{\mathrm{w}, 1}$ and high $N_{\mathrm{w}, 1}$ samples, respectively. 
Copolymer composition curve. ${ }^{\mathrm{S} 2}$ The polymerizations to obtain the precursors were performed according to the same procedures outlined in Ref. S3, with polymerization stopped at an early stage. The conversion was less than $5 \%$. We thus constructed copolymer composition curves (Figure S4). Here, HPMA and the monomer bearing the hydrazide are referred to as $\mathrm{M}_{1}$ and $\mathrm{M}_{2}$, respectively. Respective reactivity ratios $\left(r_{1}\right.$ and $r_{2}$ ) were estimated using the Fineman-Ross method. ${ }^{\mathrm{S} 4}$ The ratios were found to be $r_{1}=0.99$ and $r_{2}=0.55$, indicating that the $\mathrm{M}_{2}$ monomer is not likely to be next to another $\mathrm{M}_{2}$ monomer in the copolymer chain.

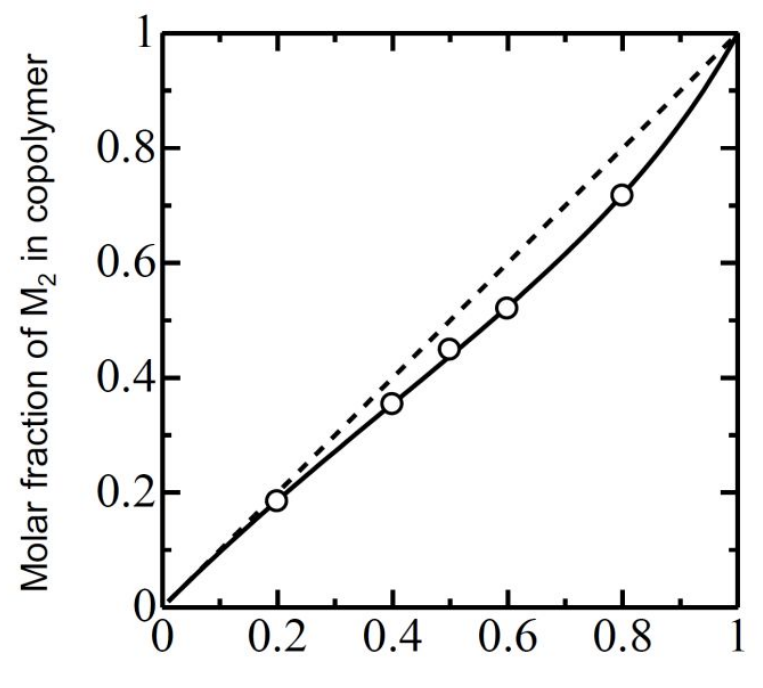

Molar fraction of $\mathrm{M}_{2}$ in monomeric feed

Figure S4. Copolymer composition curve for the precursors. ${ }^{\mathrm{S} 2}$ The points represent the experimentally obtained data, the solid curve is the fitted curves with $r_{1}=0.99$ and $r_{2}=$ 0.55 , and the broken curve is the theoretical curve with $r_{1}=r_{2}=1$. 
Electron density (contrast factor). The optical constant in X-ray scattering $\left(K_{\mathrm{e}}\right)$ is defined by $K_{\mathrm{e}}=N_{\mathrm{A}} r_{\mathrm{e}}^{2} \gamma^{2}$ where $r_{\mathrm{e}}$ is the classical electron radius, and $\gamma$ is the contrast factor calculated by

$$
\gamma=\frac{n_{\mathrm{e}}}{M_{0}}-\frac{\bar{v} \rho_{\mathrm{e}, \mathrm{solv}}}{N_{\mathrm{A}}}
$$

Here, $n_{\mathrm{e}}$ is averaged number of the electron and $M_{0}$ is the averaged molar mass of the monomer unit; i.e., $n_{\mathrm{e}} / M_{0}$ is the electron density of the polymer. $\bar{v}$ is the partial specific volume, and $\rho_{\mathrm{e}, \text { solv }}$ is the electron density of the solvent. The $\gamma$ values of the precursor and $\mathrm{P}-i$-Pyr $j$ were $0.10 \pm 0.005 \mathrm{~mol} / \mathrm{g}$, not depending on the pyrene fraction. In addition, the $n_{\mathrm{e}} /\left(M_{0} \bar{v}\right)$ value for precursor was $0.68 \mathrm{~mol} / \mathrm{cm}^{3}$, which is almost the same as the $n_{\mathrm{e}} /\left(M_{0} V\right)$ value for pyrene $\left(0.67 \mathrm{~mol} / \mathrm{cm}^{3}\right)$. Here, $V$ denotes the reciprocal of the density in the dried solid state. Therefore, the electron density of pyrene is almost the same as precursor, and there is almost no contrast between pyrene and hydrophilic parts of P-i-Pyrj.

DLS measurements. DLS measurements were performed using a DelsaMax PRO dynamic light scattering analyzer (Beckman Coulter) with an incident light wavelength of $532 \mathrm{~nm}$. Using each autocorrelation function at an angle of $163.5^{\circ}$, the relaxation time spectrum $\left[A_{\mathrm{r}}(\tau)\right]$ was obtained by a CONTIN analysis. From $A_{\mathrm{r}}(\tau)$, the hydrodynamic radius $\left(R_{\mathrm{H}}\right)$ was obtained by

$$
R_{\mathrm{H}}=\frac{k_{\mathrm{B}} T}{6 \pi \eta \sum \tau^{-1} A_{\mathrm{r}}(\tau)} \#(\mathrm{~S} 4)
$$

The $R_{\mathrm{H}}$ values for P-1-Pyr4, P-1-Pyr6, and P-1-Pyr7 were 3.7, 3.9, and $4.0 \mathrm{~nm}$, respectively. We note that the observed relaxation mode may possibly include not only 
translational diffusion but also other modes such as internal motion. To investigate the relaxation in detail, multi-angle DLS measurements should be performed. ${ }^{\text {55 }}$

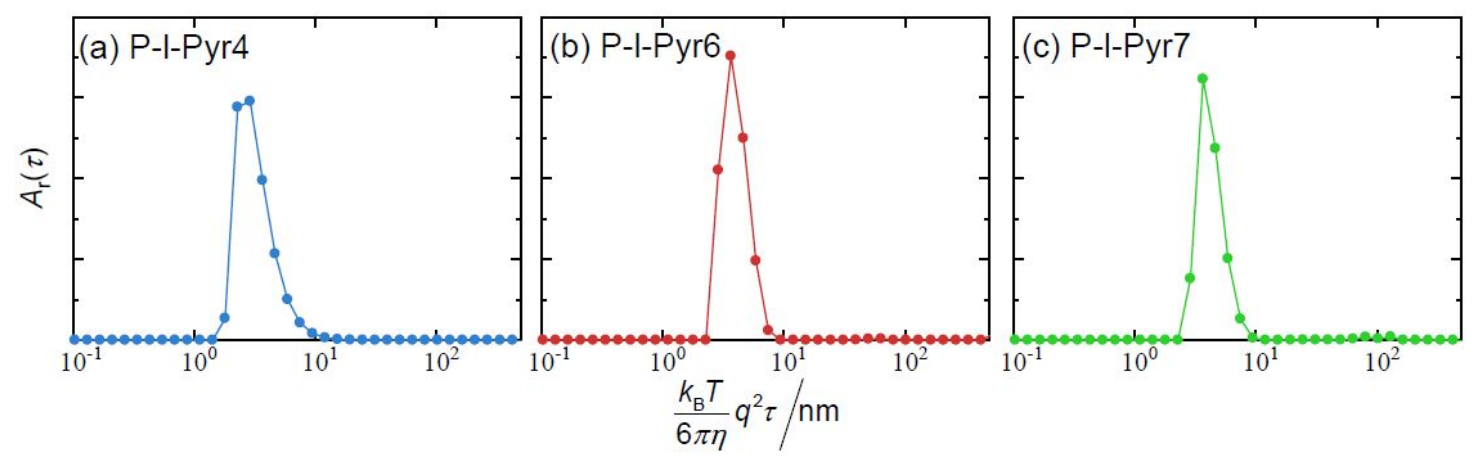

Figure S5. Relaxation time spectra of P-1-Pyr4 (a), P-1-Pyr6 (b), and P-1-Pyr7 (c) at $c=$ $5 \mathrm{mg} / \mathrm{mL}$, which were obtained by DLS measurements.

\section{REFERENCES}

(S1) Pedersen, J. S.; Laso, M.; Schurtenberger, P. Monte Carlo study of excluded volume effects in wormlike micelles and semiflexible polymers. Phys. Rev. E 1996, 54, R5917-R5920.

(S2) Doi, K; Takahashi, R.; Shota, S.; Sakurai, K. Synthesis and Dilute Solution Properties of $N$-(2-Hydroxypropyl)methacrylamide-Based Copolymers. submitted to $J$. Fiber Sci. Technol., in Japanese.

(S3) Etrych, T.; Mrkvan, T.; Chytil, P.; Koňaḱ, C.; Ř́hová, B.; Ulbrich, K. $\mathrm{N}$-(2-hydroxypropyl)methacrylamide-based polymer conjugates with $\mathrm{pH}$-controlled 
activation of doxorubicin. I. New synthesis, physicochemical characterization and preliminary biological evaluation. J. Appl. Polym. Sci. 2008, 109, 3050-3061.

(S4) Fineman, M.; Ross, S. D. Linear method for determining monomer reactivity ratios in copolymerization. J. Polym. Sci. 1950, 5, 259-265.

(S5) Chu, B. Dynamic Light Scattering, in Soft Matter Characterization, Borsali, R.; Pecora, R. Eds., Springer, 2008. 